\title{
OPEN Blood transfusions increase the risk for venous thromboembolism events following total joint arthroplasty
}

Noam Shohat ${ }^{1,2}$, Leanne Ludwick ${ }^{1}$, Graham S. Goh ${ }^{1}$, Matthew Sherman ${ }^{1}$, Joseph Paladino ${ }^{1}$ \& Javad Parvizi ${ }^{1,3}$ 凶

The association between blood transfusions and thromboembolic events (VTE) following total joint arthroplasty (TJA) remains debatable. Using contemporary institutional data, this study aimed to determine whether blood transfusions increase the risk of VTE following primary and revision TJA. This was a single institution, retrospective cohort study. The clinical records of all patients $(n=34,824)$ undergoing primary and revision TJA between 2009 and 2020 were reviewed. Demographic variables, co-morbidities, type of chemoprophylaxis and intraoperative factors such as use of tranexamic acid were collected. Clinical notes, hospital orders, and discharge summaries were reviewed to determine if a patient received a blood transfusion. Comprehensive queries utilizing keywords for VTE were conducted in clinical notes, physician dictations, and patient-provider phone-call logs. Propensity score matching as well as adjusted mixed models were performed. After adjusting for various confounders, results from regression analysis showed a significant association between allogenic blood transfusions and risk for developing VTE following primary and revision TJA (OR 4.11, 95\% $\mathrm{Cl} 2.53-6.69$ and $\mathrm{OR} 2.15,95 \% \mathrm{Cl} 1.12-4.13$, respectively). While this strong association remained significant for PE in both primary $(p<0.001)$ and revision $(p<0.001)$ matched cohorts, it was no longer statistically significant for DVT ( $p=0.802$ and $p=0.65$, respectively). These findings suggest that the risk of VTE is increased by approximately three-folds when blood transfusions are prescribed. This association was mainly due to higher symptomatic PE events which makes it even more worrisome. Surgeons should be aware of this association, revisit criteria for blood transfusions and use all means available in the perioperative period to optimize the patients and avoid transfusion.

Venous thromboembolism (VTE), including deep venous thrombosis (DVT) and pulmonary embolism (PE), is a dreadful complication associated with total joint arthroplasty (TJA). While several risk factors for the development of VTE have been established ${ }^{1,2}$, others are still widely debated ${ }^{2,3}$. One potentially modifiable risk factor that has gained attention due to its common and unrestricted use is allogeneic blood transfusion ${ }^{4-6}$.

The demand for blood products is on the rise worldwide resulting in significant financial and health related $\operatorname{costs}^{7-9}$. It is estimated that more than 400 million whole blood and red blood cell (RBC) units are transfused annually in the United States ${ }^{10}$. Physicians in general, and orthopedic surgeons in particular, tend to overprescribe blood transfusions ${ }^{11-14}$. In fact, Total hip (THA) and knee (TKA) arthroplasties represent the number one reason for allogeneic blood transfusion in patients undergoing elective surgery, accounting for nearly $10 \%$ of all hospital transfused red blood cell units ${ }^{12,13}$. Often times the amount of blood transfused, does not clearly correlate with the blood volume that was lost during surgery, and is often times to the detriment of patient health ${ }^{15,16}$.

Several attempts were made to try and shed light on whether blood transfusions lead to increased risk for VTE. A review of the current literature shows that registry data has consistently demonstrated an association between blood transfusions and VTE. However, all published studies to date have utilized the same National Surgical Quality Improvement Program (NSQIP) database ${ }^{4,5}$, rendering them susceptible to inherent limitations associated with registry data ${ }^{17,18}$. By contrast, several institutional studies, including one published from our institution, have failed to demonstrate a clear independent association ${ }^{6,19}$. However, these studies included

${ }^{1}$ Rothman Orthopaedic Institute at Thomas Jefferson University, Philadelphia, PA, USA. ${ }^{2}$ Sackler Faculty of Medicine, Tel Aviv University, Ramat Aviv, Israel. ${ }^{3}$ Rothman Orthopaedic Institute, 125 S 9th St. Ste 1000, Philadelphia, PA 19107, USA. ${ }^{\circledR}$ email: research@rothmanortho.com 


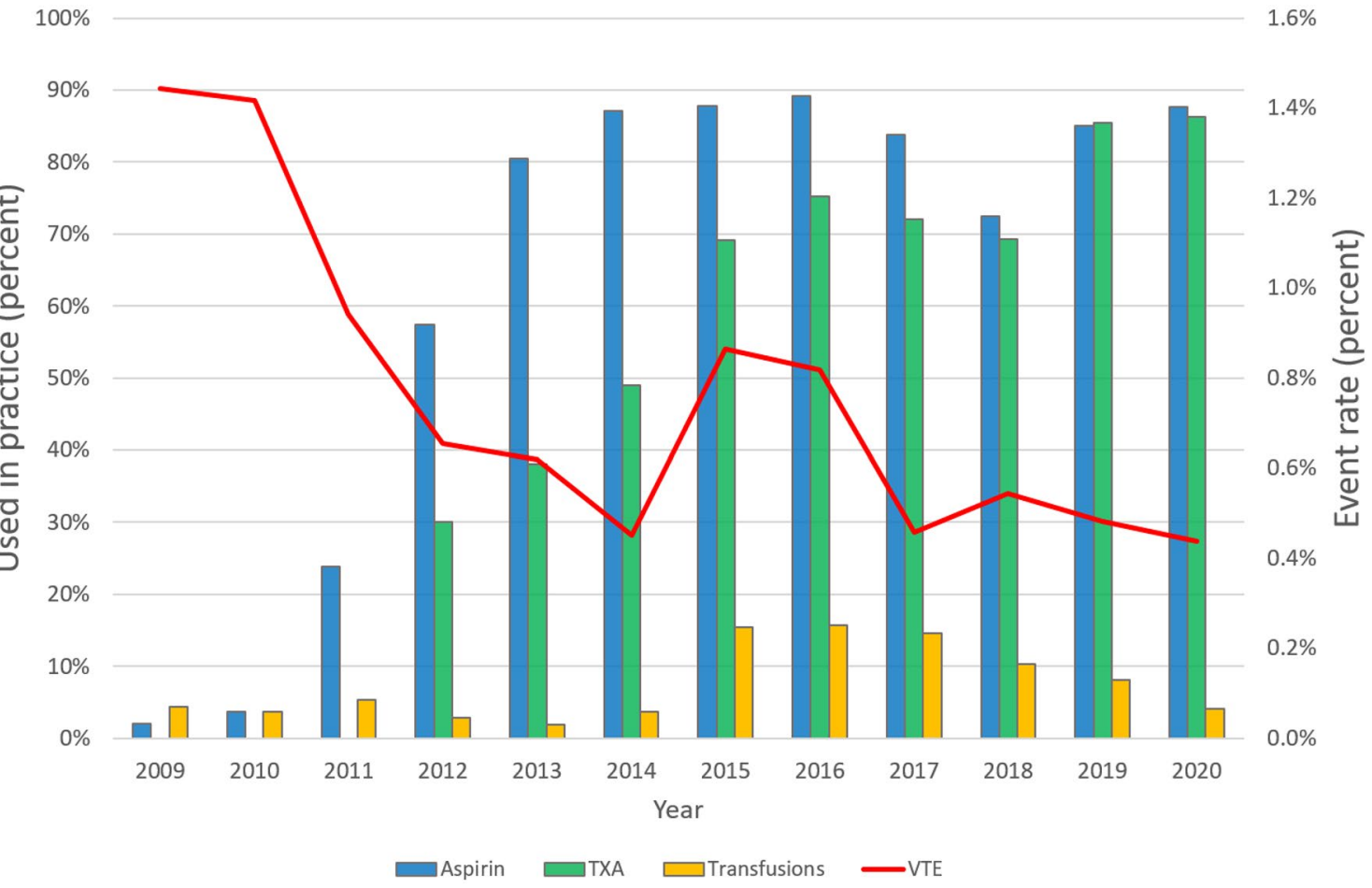

Figure 1. Relevant changes in practice over the course of the study. Bars reflect changes in aspirin and tranexamic usage as well as transfusion rates. The line represents symptomatic venous thromboembolism rates.

only primary TJA, they suffered from sample size limitations and failed to account for the change in practice patterns that have been introduced over the past decade, such as the use of tranexamic acid (TXA) and transition to aspirin as the main chemoprophylaxis agent in TJA. Given the recent advances in perioperative care as well as the lack of consensus in the literature, an updated investigation of the impact of blood transfusions on the risk of VTE following primary and revision TJA is therefore warranted.

Using contemporary institutional data, the aim of this study was to assess whether blood transfusion increases the risk of VTE following primary and revision TJA.

\section{Methods}

A single institution, retrospective cohort study was conducted following approval by the institutional review board of Thomas Jefferson University with a waiver of informed consent. This study was performed in accordance with relevant guidelines and regulations. The clinical records of 39,948 consecutive patients who underwent a primary or revision THA or TKA from January 2009 to October 2020 were reviewed. Strengthening the reporting of observational studies in epidemiology (STROBE) reporting guidelines were followed throughout the data collection process ${ }^{20}$. Patients for whom transfusion data was not available were excluded. The final cohort consisted of 34,824 patients with a minimum follow-up of 90 days.

Age, sex, race, body mass index (BMI), Charlson Comorbidity Index (CCI), Elixhauser Comorbidity Index (ECI), and American Society of Anesthesiologists (ASA) classification were collected through hospital medical records and International Classification of Diseases (ICD) codes. Clinical notes, hospital orders, and discharge summaries were reviewed to record the type of VTE prophylaxis prescribed to each patient postoperatively. Operative details including the specific joint operated on, laterality, operative time, use of cement, TXA administration, and intraoperative blood transfusions were also collected from operative reports and anesthesia case logs.

Over the course of this study, our institution underwent a transition from the use of warfarin to aspirin as the main method for chemoprophylaxis of VTE. Warfarin remained the treatment of choice for high risk patients ${ }^{2}$. Patients receiving warfarin had their INR monitored by either their personal physician or a warfarin clinic. Patients receiving aspirin were either administered regular dose $(325 \mathrm{mg})$ or low dose $(81 \mathrm{mg})$ twice daily. VTE prophylaxis with either agent was continued for 4 to 6 weeks postoperatively. Additional relevant change in practice during the course of the study included transition to routine use of tranexamic acid infusion during surgery (Fig. 1).

The main exposure variable was the use of allogeneic blood transfusion (packed red blood cells). This was divided into 3 distinct categories: no transfusion, 1 transfusion unit and 2 or more transfusion units. The latter two categories were grouped together for the main analysis, but analyzed individually as a subgroup in adjusted models. The primary outcome was the first occurrence of a symptomatic DVT or PE within 90 days of surgery. Symptomatic VTE occurring within 90 days of the operation were identified from medical records through ICD 9 and 10 codes (Supplementary Table S1). To enhance the capture rate, comprehensive queries utilizing keywords for DVT and PE were conducted in clinical notes, physician dictations, and patient-provider phone-call logs 


\begin{tabular}{|c|c|c|c|}
\hline & No transfusion $(n=28,529)$ & Any transfusion $(\mathrm{n}=956)$ & P-value \\
\hline \multicolumn{4}{|l|}{ Demographics } \\
\hline Age, year (mean,SD) & $63.7(10.5)$ & $67.9(12.4)$ & $<0.001$ \\
\hline Sex, female & $15,491(54.3 \%)$ & $559(58.5 \%)$ & 0.012 \\
\hline BMI, Kg/M² (mean,SD) & $29.9(5.36)$ & $28.9(5.74)$ & $<0.001$ \\
\hline \multicolumn{4}{|l|}{ Co-morbidities } \\
\hline CCI (mean,SD) & $0.39(0.84)$ & $1.16(1.67)$ & $<0.001$ \\
\hline History of VTE & $926(3.25 \%)$ & $77(8.05 \%)$ & $<0.001$ \\
\hline CHF & $455(1.59 \%)$ & $95(9.94 \%)$ & $<0.001$ \\
\hline CPD & $3157(11.1 \%)$ & $171(17.9 \%)$ & $<0.001$ \\
\hline CVD & $318(1.11 \%)$ & $44(4.60 \%)$ & $<0.001$ \\
\hline Dementia & $42(0.15 \%)$ & $6(0.63 \%)$ & 0.004 \\
\hline $\mathrm{DM}$ & $1390(4.87 \%)$ & $93(9.73 \%)$ & $<0.001$ \\
\hline Hemiparesis & $15(0.05 \%)$ & $2(0.21 \%)$ & 0.104 \\
\hline \multicolumn{4}{|l|}{ Malignancy } \\
\hline Non-metastatic & $243(0.85 \%)$ & $26(2.72 \%)$ & $<0.001$ \\
\hline Metastatic & $41(0.14 \%)$ & $20(2.09 \%)$ & \\
\hline MI & $965(3.38 \%)$ & $65(6.80 \%)$ & $<0.001$ \\
\hline \multicolumn{4}{|l|}{ Liver disease } \\
\hline Mild & $265(0.93 \%)$ & $25(2.62 \%)$ & $<0.001$ \\
\hline Moderate/severe & $11(0.04 \%)$ & $6(0.63 \%)$ & $<0.001$ \\
\hline PUD & $82(0.29 \%)$ & $23(2.41 \%)$ & $<0.001$ \\
\hline PVD & $388(1.36 \%)$ & $41(4.29 \%)$ & $<0.001$ \\
\hline Renal failure & $571(2.00 \%)$ & $107(11.2 \%)$ & $<0.001$ \\
\hline Rheumatic disease & $856(3.00 \%)$ & $55(5.75 \%)$ & $<0.001$ \\
\hline \multicolumn{4}{|l|}{ Operative factors } \\
\hline Joint, knee & $13,750(48.2 \%)$ & $439(45.9 \%)$ & 0.176 \\
\hline Bilateral surgery & $1724(6.04 \%)$ & $95(9.94 \%)$ & $<0.001$ \\
\hline Tranexamic acid & $13,380(46.9 \%)$ & $386(40.4 \%)$ & $<0.001$ \\
\hline Operative time (mean,SD) & $72.2(26.6)$ & $98.5(55.5)$ & $<0.001$ \\
\hline Cement used & $11,273(39.5 \%)$ & $403(42.2 \%)$ & 0.108 \\
\hline \multicolumn{4}{|l|}{ Anticoagulation } \\
\hline Aspirin & $17,653(61.9 \%)$ & $598(62.6 \%)$ & 0.697 \\
\hline Other & $10,876(38.1 \%)$ & $358(37.4 \%)$ & \\
\hline Symptomatic VTE & $198(0.69 \%)$ & $31(3.24 \%)$ & $<0.001$ \\
\hline Pulmonary Emboli & $122(0.43 \%)$ & $25(2.62 \%)$ & $<0.001$ \\
\hline Deep Vein Thrombosis & $96(0.34 \%)$ & $9(0.94 \%)$ & 0.007 \\
\hline
\end{tabular}

Table 1. Patient characteristics, comorbidities, operative factors and type of VTE prophylaxis for the primary TJA cohort and their VTE rates. BMI Body Mass Index, $K g$ kilogram, $M$ meter, CCI Charlson Comorbidity Index, $C H F$ congestive heart failure, $C P D$ cardiopulmonary disease, $C V D$ cardiovascular disease, $D M$ diabetes mellitus, $M I$ myocardial infraction, $P U D$ peptic ulcer disease, $P V D$ peripheral vascular disease, $V T E$ venous thromboembolism event.

(Supplementary Table S2). Notes containing a keyword for DVT $(n=44,752)$ and PE $(n=14,878)$ were isolated and manually reviewed. All readmissions within 90 days were also reviewed to detect any uncaptured VTE event. Patients that had a documented diagnosis, confirmatory study (ultrasound for DVT or CT scan for PE), or received treatment for VTE were considered to have met the primary endpoint. For events that were diagnosed in an outside facility for which an objectively confirmed study was not readily available to review, diagnosis relied on a documented note reporting a confirmatory study as well as a symptomatic event that received treatment. To avoid inclusion of clinically insignificant clots (unimportant or small, possibly muscle vein clots), cases were excluded if they were specifically documented as asymptomatic or not mandating anticoagulation treatment.

R Studio (version 3.6.3, Vienna, Austria) was used for the statistical analyses ${ }^{21}$. The level of statistical significance was defined as a p-value of less than under 0.05 . To minimize the loss of power and decrease bias owing to exclusion of cases that were missing 1 or more covariates, missing covariates were imputed 10 times using the multivariate sequential regression approach. First, univariate comparisons between patients who did not receive blood transfusion compared to those who received one or more transfusions were performed, using chi-square for dichotomous variables, and T-test for continuous variables. Second, an unadjusted followed by adjusted logistic regression analysis looking at mixed models were performed with VTE as the dependent outcome. For these analyses, blood transfusion was broken down into three distinct categories (no transfusion, 1 transfusion, 


\begin{tabular}{|c|c|c|c|}
\hline & No transfusion $(n=4232)$ & Any transfusion $(n=1107)$ & P-value \\
\hline \multicolumn{4}{|l|}{ Demographics } \\
\hline Age, year (mean,SD) & $63.0(12.8)$ & $66.5(12.5)$ & $<0.001$ \\
\hline Sex, female & $2169(51.3 \%)$ & $595(53.7 \%)$ & 0.148 \\
\hline BMI, Kg/M² (mean,SD) & $30.1(6.18)$ & $30.4(6.90)$ & 0.422 \\
\hline \multicolumn{4}{|l|}{ Co-morbidities } \\
\hline CCI $($ mean, SD) & $0.55(1.10)$ & $1.16(1.62)$ & $<0.001$ \\
\hline History of VTE & $284(6.71 \%)$ & $102(9.21 \%)$ & 0.005 \\
\hline CHF & $125(2.95 \%)$ & $101(9.12 \%)$ & $<0.001$ \\
\hline $\mathrm{CPD}$ & $524(12.4 \%)$ & $205(18.5 \%)$ & $<0.001$ \\
\hline CVD & $66(1.56 \%)$ & $49(4.43 \%)$ & $<0.001$ \\
\hline Dementia & $6(0.14 \%)$ & $26(2.35 \%)$ & $<0.001$ \\
\hline $\mathrm{DM}$ & $169(3.99 \%)$ & $82(7.41 \%)$ & $<0.001$ \\
\hline Hemiparesis & $8(0.19 \%)$ & $6(0.54 \%)$ & 0.051 \\
\hline \multicolumn{4}{|l|}{ Malignancy } \\
\hline Non-metastatic & $58(1.37 \%)$ & $22(1.99 \%)$ & 0.069 \\
\hline Metastatic & $28(0.66 \%)$ & $13(1.17 \%)$ & \\
\hline MI & $196(4.63 \%)$ & $89(8.04 \%)$ & $<0.001$ \\
\hline \multicolumn{4}{|l|}{ Liver disease } \\
\hline Mild & $32(0.76 \%)$ & $25(2.26 \%)$ & $<0.001$ \\
\hline Moderate/severe & $1(0.02 \%)$ & $10(0.90 \%)$ & $<0.001$ \\
\hline PUD & $17(0.40 \%)$ & $9(0.81 \%)$ & 0.132 \\
\hline PVD & $69(1.63 \%)$ & $46(4.16 \%)$ & $<0.001$ \\
\hline Renal failure & $158(3.73 \%)$ & $142(12.8 \%)$ & $<0.001$ \\
\hline Rheumatic disease & $217(5.13 \%)$ & $86(7.77 \%)$ & $<0.001$ \\
\hline \multicolumn{4}{|l|}{ Operative factors } \\
\hline Joint, knee & $1757(41.5 \%)$ & $353(31.9 \%)$ & $<0.001$ \\
\hline Bilateral surgery & $40(0.95 \%)$ & $12(1.08 \%)$ & 0.805 \\
\hline Tranexamic acid & $1224(28.9 \%)$ & $360(32.5 \%)$ & 0.022 \\
\hline Operative time (mean,SD) & $120(50.0)$ & $149(75.6)$ & $<0.001$ \\
\hline Cement used & $1840(43.5 \%)$ & $478(43.2 \%)$ & 0.885 \\
\hline \multicolumn{4}{|l|}{ Anticoagulation } \\
\hline Aspirin & $1790(42.3 \%)$ & $553(50.0 \%)$ & $<0.001$ \\
\hline Other & $2442(57.7 \%)$ & $554(50.0 \%)$ & \\
\hline Symptomatic VTE & $49(1.16 \%)$ & $37(3.34 \%)$ & $<0.001$ \\
\hline Pulmonary emboli & $8(0.19 \%)$ & $12(1.08 \%)$ & $<0.001$ \\
\hline Deep vein thrombosis & $43(1.02 \%)$ & $28(2.53 \%)$ & $<0.001$ \\
\hline
\end{tabular}

Table 2. Patient characteristics, comorbidities, operative factors and type of VTE prophylaxis for the revision TJA cohort and their VTE rates. BMI Body Mass Index, Kg kilogram, M meter, CCI Charlson Comorbidity Index, $C H F$ congestive heart failure, $C P D$ cardiopulmonary disease, $C V D$ cardiovascular disease, $D M$ diabetes mellitus, $M I$ myocardial infraction, $P U D$ peptic ulcer disease, $P V D$ peripheral vascular disease, $V T E$ venous thromboembolism event.

and 2 or more transfusions). Significant outcomes in the univariate that were included in the regression analysis included blood transfusion, age, BMI, sex, ASA score, CCI score, joint, unilateral vs. bilateral, and use of TXA. The patient identification number was used as the random term to account for patients who underwent multiple procedures to avoid bias from unmeasurable risk factors. Third, to account for significant differences between the non-transfusion and transfusion groups, the groups were matched for age, BMI, sex, all comorbidities in the cohort (see Table 1), joint, unilateral vs. bilateral, primary vs. revision, as well as the use of cement and TXA using propensity score matching.

\section{Results}

Of the 34,824 patients that were eligible for analysis, 2063 (5.9\%) received one or more units blood transfusions. There were statistically significant differences between the non-transfusion and transfusion groups in both the primary and revision cohort. Patients who received a blood transfusion were older and had more comorbidities and being older. There were also marked differences in operative factors between the 2 groups (Tables 1 and 2).

Primary TJA. In the unmatched cohort, the rate of symptomatic VTE was $0.69 \%(198 / 28,529)$ in the nontransfusion group compared to $3.24 \%(31 / 956)$ in the transfusion group $(\mathrm{p}<0.001)$. This association remained 

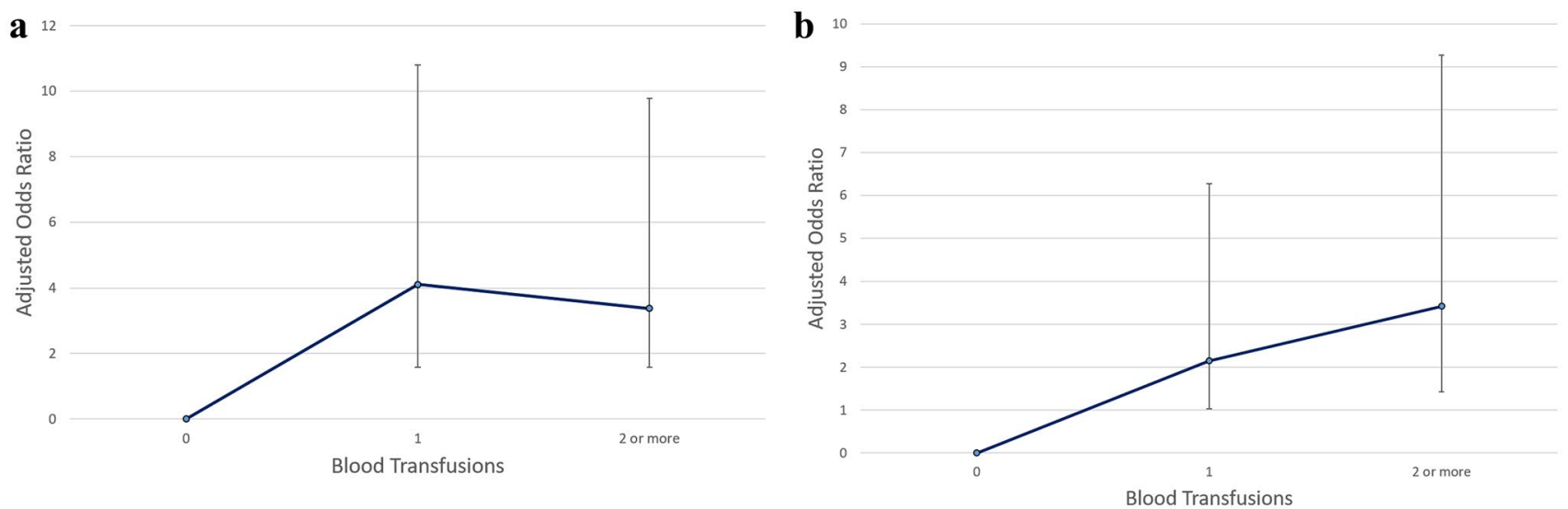

Figure 2. Adjusted odds ratios (ORs) of symptomatic venous thromboembolism with increased number of transfusions versus no transfusion in the primary cohort (a) and revision cohort (b). ${ }^{\star}$ Bars reflecting $95 \%$ confidence interval.

\begin{tabular}{|c|c|c|c|c|c|}
\hline Variable & Estimate & Odds ratio & Lower 95 & Upper 95 & P Value \\
\hline \multicolumn{6}{|c|}{ Blood transfusion } \\
\hline 1 & 1.41 & 4.11 & 2.53 & 6.69 & $<0.001$ \\
\hline 2 or more & 1.22 & 3.38 & 1.79 & 6.39 & $<0.001$ \\
\hline Age & 0.02 & 1.02 & 1.01 & 1.04 & 0.001 \\
\hline $\mathrm{BMI}, \mathrm{Kg} / \mathrm{M}^{2}$ & 0.05 & 1.05 & 1.03 & 1.07 & $<0.001$ \\
\hline Male & 0.25 & 1.29 & 0.997 & 1.66 & 0.053 \\
\hline Aspirin & -0.43 & 0.65 & 0.48 & 0.89 & 0.006 \\
\hline CCI & 0.10 & 1.11 & 0.997 & 1.24 & 0.056 \\
\hline Hip & -0.74 & 0.48 & 0.36 & 0.64 & $<0.001$ \\
\hline Bilateral & 0.75 & 2.11 & 1.44 & 3.11 & $<0.001$ \\
\hline TXA & -0.33 & 0.72 & 0.51 & 1.01 & 0.056 \\
\hline Op time & 0.002 & 1.002 & 0.998 & 1.01 & 0.307 \\
\hline
\end{tabular}

Table 3. Logistic mixed model regression evaluating the association between 1 blood transfusion, or 2 or more (with zero transfusions as the reference) and risk for VTE in the primary TJA cohort. BMI Body Mass Index, $K g$ kilogram, $M$ meter, CCI Charlson Comorbidity Index, TXA tranexamic acid.

significant for both PE and DVT separately (Table 1). Regression analysis showed a significant association between 1 blood transfusion (OR 4.11, 95\% CI 2.53-6.69, p < 0.001) and 2 or more blood transfusions (OR 3.38, 95\% CI 1.79-6.39, p < 0.001) with risk for developing VTE (Fig. 2a). Others factors that were significantly associated with VTE in the regression analysis included older age, higher BMI, TKA (as opposed to THA), bilateral arthroplasties, and non-aspirin prophylaxis (Table 3).

The propensity score matched cohort comprised 956 patients per group. Both groups showed no significant difference in demographic variables, comorbidities, operative factors or chemoprophylaxis medications (Table 4). In this matched cohort, the association between blood transfusions with the risk for developing VTE remained significant $(\mathrm{p}=0.002)$ and was 3 times higher in patients who received blood transfusion $(3.24 \%)$ compared to those who did not (1.05\%). While this association remained significant for PE $(2.62 \%$ vs. $0.31 \%, \mathrm{p}<0.001)$ it was no longer statistically significant for DVT $(0.94 \%$ vs. $0.73 \%, \mathrm{p}=0.802)$.

Revision TJA. In the unmatched cohort, the rate of symptomatic VTE was $1.16 \%(49 / 4232)$ in the nontransfusion group compared to $3.34 \%(37 / 1107)$ in the transfusion group $(\mathrm{p}<0.001)$. This association remained significant for both PE and DVT separately (Table 2). Regression analysis showed a significant association between 1 blood transfusion (OR 2.15, 95\% CI 1.12-4.13, $\mathrm{p}=0.021)$ and 2 or more blood transfusions (OR 3.42, 95\% CI 1.99-5.85, p<0.001) with risk for developing VTE (Fig. 2b). The only others factor that were significantly associated with VTE in the regression analysis was non-aspirin prophylaxis (Table 5).

The propensity score matched cohort comprised 1107 patients per group. The only factors that remained significantly different between the 2 groups after matching were dementia and liver disease (Table 6). In this matched cohort, the association between blood transfusions with the risk for developing VTE remained significant $(\mathrm{p}=0.003)$ and was 2.5 times higher in patients who received blood transfusion $(3.34 \%)$ compared to those who did not (1.36\%). While this association remained significant for PE $(1.08 \%$ vs. $0.09 \%, \mathrm{p}=0.005)$ it was no longer statistically significant for DVT ( $1.36 \%$ vs. $2.53 \%, \mathrm{p}=0.065)$. 


\begin{tabular}{|c|c|c|c|}
\hline & No transfusion $(n=956)$ & Any transfusion $(n=956)$ & P-value \\
\hline \multicolumn{4}{|l|}{ Demographics and habits } \\
\hline Age, year (mean, SD) & $68.4(10.3)$ & $67.9(12.4)$ & 0.980 \\
\hline Sex, female & $539(56.4 \%)$ & $559(58.5 \%)$ & 0.380 \\
\hline BMI, Kg/M² (mean, SD) & $28.8(5.19)$ & $28.9(5.74)$ & 0.955 \\
\hline \multicolumn{4}{|l|}{ Co-morbidities } \\
\hline CCI $($ mean, SD) & $1.25(1.81)$ & $1.16(1.67)$ & 0.533 \\
\hline History of VTE & $79(8.26 \%)$ & $77(8.05 \%)$ & 0.933 \\
\hline $\mathrm{CHF}$ & $108(11.3 \%)$ & $95(9.94 \%)$ & 0.373 \\
\hline CPD & $184(19.2 \%)$ & $171(17.9 \%)$ & 0.480 \\
\hline CVD & $54(5.65 \%)$ & $44(4.60 \%)$ & 0.351 \\
\hline Dementia & $5(0.52 \%)$ & $6(0.63 \%)$ & 1.000 \\
\hline $\mathrm{DM}$ & $112(11.7 \%)$ & $93(9.73 \%)$ & 0.183 \\
\hline Hemiparesis & $2(0.21 \%)$ & $2(0.21 \%)$ & 1.000 \\
\hline \multicolumn{4}{|l|}{ Malignancy } \\
\hline Non-metastatic & $30(3.14 \%)$ & $26(2.72 \%)$ & 0.863 \\
\hline Metastatic & $20(2.09 \%)$ & $20(2.09 \%)$ & \\
\hline MI & $76(7.95 \%)$ & $65(6.80 \%)$ & 0.382 \\
\hline \multicolumn{4}{|l|}{ Liver disease } \\
\hline Mild & $22(2.30 \%)$ & $25(2.62 \%)$ & 0.768 \\
\hline Moderate/severe & $6(0.63 \%)$ & $6(0.63 \%)$ & 1.000 \\
\hline PUD & $17(1.78 \%)$ & $23(2.41 \%)$ & 0.424 \\
\hline PVD & $47(4.92 \%)$ & $41(4.29 \%)$ & 0.585 \\
\hline Renal failure & $104(10.9 \%)$ & $107(11.2 \%)$ & 0.884 \\
\hline Rheumatic disease & $50(5.23 \%)$ & $55(5.75 \%)$ & 0.688 \\
\hline \multicolumn{4}{|l|}{ Operative factors } \\
\hline Joint, knee & $443(46.3 \%)$ & $439(45.9 \%)$ & 0.891 \\
\hline Bilateral surgery & $109(11.4 \%)$ & $95(9.94 \%)$ & 0.336 \\
\hline Tranexamic acid & $380(39.7 \%)$ & $386(40.4 \%)$ & 0.815 \\
\hline Operative time (mean, SD) & $93.9(55.0)$ & $98.5(55.5)$ & 0.074 \\
\hline Cement used & $397(41.5 \%)$ & $403(42.2 \%)$ & 0.815 \\
\hline \multicolumn{4}{|l|}{ Anticoagulation } \\
\hline Aspirin & $611(63.9 \%)$ & $598(62.6 \%)$ & 0.569 \\
\hline Other & $345(36.1 \%)$ & $358(37.4 \%)$ & \\
\hline Symptomatic VTE & $10(1.05 \%)$ & $31(3.24 \%)$ & 0.002 \\
\hline Pulmonary emboli & $3(0.31 \%)$ & $25(2.62 \%)$ & $<0.001$ \\
\hline Deep vein thrombosis & $7(0.73 \%)$ & $9(0.94 \%)$ & 0.802 \\
\hline
\end{tabular}

Table 4. Patient characteristics, comorbidities, operative factors and type of VTE prophylaxis for the matched primary TJA cohort and VTE rates. BMI Body Mass Index, $K g$ kilogram, $M$ meter, CCI Charlson Comorbidity Index, $C H F$ congestive heart failure, $C P D$ cardiopulmonary disease, $C V D$ cardiovascular disease, $D M$ diabetes mellitus, $M I$ myocardial infraction, $P U D$ peptic ulcer disease, $P V D$ peripheral vascular disease, $V T E$ venous thromboembolism event. ${ }^{*}$ The groups were matched for age, BMI, sex, all comorbidities in the cohort (see Table 1), joint, unilateral vs. bilateral, as well as the use of cement and TXA.

\section{Discussion}

This is the largest single institution study to date to evaluate the association between blood transfusions and the risk for subsequent symptomatic VTE following primary and revision TJA. Using a contemporary cohort to account for major advances in perioperative care over the past decade, our findings suggest that an independent, dose-dependent association between allogeneic blood transfusions and the risk for subsequent postoperative VTE exists. Given the three-fold increase in VTE risk following a blood transfusion, the present findings help to raise awareness about this important association and underscore the need for stringent perioperative protocols to ration the use of blood transfusions following TJA.

The biological mechanisms substantiating the increased thrombotic risk following blood transfusion have been well described ${ }^{22,23}$. It is important to acknowledge that increased blood viscosity is an established risk factor for thrombosis and the shape, deformability, and viscoelastic properties of red blood cells make them the primary determinants of viscosity ${ }^{24-26}$. Red blood cells also bind to fibrinogen, and this interaction is a key mediator of blood viscosity ${ }^{26}$. In vitro and mouse models imply direct and indirect complex biochemical mechanisms by which red blood cells contribute to thrombus initiation and propagation ${ }^{27,28}$. One suggested mechanism is through modulation of the inflammatory cascade ${ }^{29}$. This could result from a direct immune response to the blood 


\begin{tabular}{|l|r|l|l|l|r|}
\hline Variable & Estimate & Odds ratio & Lower 95 & Upper 95 & P value \\
\hline \multicolumn{5}{|l|}{ Blood transfusion } \\
\hline 1 & 0.77 & 2.15 & 1.12 & 4.13 & 0.021 \\
\hline 2 or more & 1.23 & 3.42 & 1.99 & 5.85 & $<0.001$ \\
\hline Age & 0.02 & 1.02 & 0.998 & 1.04 & 0.070 \\
\hline BMI, Kg/M & 0.01 & 1.01 & 0.98 & 1.04 & 0.578 \\
\hline Male & 0.27 & 1.31 & 0.85 & 2.02 & 0.221 \\
\hline Aspirin & -1.40 & 0.25 & 0.13 & 0.48 & $<0.001$ \\
\hline CCI & 0.08 & 1.08 & 0.95 & 1.23 & 0.216 \\
\hline Hip & -0.37 & 0.69 & 0.44 & 1.08 & 0.105 \\
\hline TXA & -0.43 & 0.65 & 0.32 & 1.34 & 0.244 \\
\hline Op time & 0.001 & 1.00 & 0.997 & 1.004 & 0.617 \\
\hline
\end{tabular}

Table 5. Logistic mixed model regression evaluating the association between 1 blood transfusion, or 2 or more (with zero transfusions as the reference) and risk for VTE in the revision TJA cohort. BMI Body Mass Index, $\mathrm{Kg}$ kilogram, $M$ meter, CCI Charlson Comorbidity Index, TXA tranexamic acid.

transfusion or due to oxidative stress related to the storage of red blood cells and consequently surface damage resulting in a pro-coagulative state ${ }^{27,30}$. Other studies suggest platelet over activity as a possible causative factor ${ }^{23}$. Elevated hematocrit increases the interaction and adhesion of platelets to thrombi and platelets isolated from RBC-transfused patients show significantly enhanced aggregation ${ }^{31}$. This growing body of molecular evidence corresponds to the clinical findings of our study.

Recently published registry data, all from the NSQIP database, have suggested an association between blood transfusions and VTE following orthopedic procedures ${ }^{4,5}$. Goel et al. analyzed 750,937 patients from this database, of which 153,320 underwent orthopedic surgery. The results of their subgroup analysis showed an adjusted OR of 1.7 (95\% CI 1.5-2.0) for developing VTE following blood transfusion. Consistent with these findings, Acuña et al. utilized the same database and timeframe to examine the association in 333,463 patients undergoing $\mathrm{TKA}^{5}$. While they found an initial association between perioperative blood transfusions and DVT (adjusted OR $1.32,95 \%$ CI 1.14-1.53), their propensity score model failed to confirm this association. Notwithstanding, major limitations of the abovementioned studies that are common to registry-based data are the inaccuracies in data input as well as inability to control for inherent biases arising from wide practice variations and unmeasurable confounders $^{17,18}$. While they attempted to adjust for demographic and co-morbidities, other confounders that have been suggested to play major role in the development of VTE, such as a history of previous VTE, operative factors, type of chemoprophylaxis and other contemporary changes in practice including the use of TXA could not be accounted for as these variables are not well documented in national registries. Using contemporary data derived from a single institution to circumvent the inherent biases from unmeasurable confounders, the present findings echo that of previous studies. All efforts were made to isolate and determine the independent association between blood transfusion and VTE using adjusted models and propensity score matching, and our results reinforce the notion that blood transfusions increase the risk for VTE following both primary and revision TJA. Propensity score analysis results suggested that this association is mainly true for PE events as the association with DVT lost statistical significance in the matched cohort.

The current findings were contradictory to those published from our own institution previously ${ }^{6}$, which is a testament to the immense difficulty in isolating a single variable association when the event rate is low and many confounding variables exist. While the study by Jackson et al. evaluated a large number of 29,000 TJA, the event rate was only $1.04 \%$ (the number of VTEs in the transfused group), possibly rendering the study underpowered to detect an effect size that may be still clinically meaningful. This limitation was further highlighted when an admirable attempt was made to adjust for a large number of variables based on a validated VTE risk score, which may in turn have resulted in overadjustment ${ }^{32}$. Another plausible reason for the discrepancy between our two studies could be the difference in time period between the study cohorts. Jackson et al. included all primary TJA patients operated since 2000 and did not include the last 6 years of practice in their analysis. Consequently, only $20 \%$ of our cohort had overlapped with that of the previous study. Changes in the perioperative care including early mobility, the use of TXA, more aggressive rehabilitation, same-day discharge, and transition to aspirin as the chemoprophylactic agent of choice could not be accounted for in the previous publication and may have confounded the results as well.

The annual total units of blood transfused in U.S. hospitals has increased from 15 to 21 million in a short span of 5 years ${ }^{33}$. Frank et al. examined the medical records of more than 48,000 surgical patients over an 18-month period and found that 2,981 patients (6.2 percent) were given blood transfusions during surgery ${ }^{13}$. Interestingly, the authors noted wide variations in blood transfusion prescribing patterns among surgeons and anesthesiologists. For instance, patients undergoing cardiac surgeries received blood at much lower trigger points compared to patients having other surgeries, whereas patients undergoing orthopedic surgery received blood at higher trigger points (often at or above $10 \mathrm{~g}$ per deciliter). Moreover, the amount of blood transfused did not correlate the clinical status of the patient or the degree of blood loss typically expected during each type of surgery ${ }^{15,16}$. In addition, orthopedic surgeons in particular have been shown to overprescribe blood transfusions compared to other specialties ${ }^{11-14}$. The first step in dealing with a problem is acknowledging we have one. As such, the results 


\begin{tabular}{|c|c|c|c|}
\hline & No transfusion $(n=1107)$ & Any transfusion $(n=1107)$ & P-value \\
\hline \multicolumn{4}{|l|}{ Demographics and habits } \\
\hline Age, year (mean, SD) & $66.7(11.4)$ & $66.5(12.5)$ & 0.782 \\
\hline Sex, female & $593(53.6 \%)$ & $595(53.7 \%)$ & 0.966 \\
\hline BMI, Kg/M² (mean, SD) & $30.7(6.65)$ & $30.4(6.90)$ & 0.217 \\
\hline \multicolumn{4}{|l|}{ Co-morbidities } \\
\hline CCI (mean, SD) & $1.10(1.53)$ & $1.16(1.62)$ & 0.499 \\
\hline History of VTE & $100(9.03 \%)$ & $102(9.21 \%)$ & 0.941 \\
\hline $\mathrm{CHF}$ & $91(8.22 \%)$ & $101(9.12 \%)$ & 0.497 \\
\hline $\mathrm{CPD}$ & $214(19.3 \%)$ & $205(18.5 \%)$ & 0.664 \\
\hline CVD & $39(3.52 \%)$ & $49(4.43 \%)$ & 0.328 \\
\hline Dementia & $6(0.54 \%)$ & $26(2.35 \%)$ & 0.001 \\
\hline $\mathrm{DM}$ & $80(7.23 \%)$ & $82(7.41 \%)$ & 0.935 \\
\hline Hemiparesis & $80(7.23 \%)$ & $82(7.41 \%)$ & 0.751 \\
\hline \multicolumn{4}{|l|}{ Malignancy } \\
\hline Non-metastatic & $24(2.17 \%)$ & $22(1.99 \%)$ & 0.727 \\
\hline Metastatic & $17(1.54 \%)$ & $13(1.17 \%)$ & \\
\hline MI & $81(7.32 \%)$ & $89(8.04 \%)$ & 0.576 \\
\hline \multicolumn{4}{|l|}{ Liver disease } \\
\hline Mild & $24(2.17 \%)$ & $25(2.26 \%)$ & 1.000 \\
\hline Moderate/severe & $1(0.09 \%)$ & $10(0.90 \%)$ & 0.016 \\
\hline PUD & $7(0.63 \%)$ & $9(0.81 \%)$ & 0.802 \\
\hline PVD & $35(3.16 \%)$ & $46(4.16 \%)$ & 0.258 \\
\hline Renal failure & $127(11.5 \%)$ & $142(12.8 \%)$ & 0.362 \\
\hline Rheumatic disease & $100(9.03 \%)$ & $86(7.77 \%)$ & 0.319 \\
\hline \multicolumn{4}{|l|}{ Operative factors } \\
\hline Joint, knee & $392(35.4 \%)$ & $353(31.9 \%)$ & 0.087 \\
\hline Bilateral surgery & $14(1.26 \%)$ & $12(1.08 \%)$ & 0.844 \\
\hline Tranexamic acid & $392(35.4 \%)$ & $360(32.5 \%)$ & 0.164 \\
\hline Operative time (mean,SD) & $144(64.6)$ & $149(75.6)$ & 0.381 \\
\hline Cement used & $513(46.3 \%)$ & $478(43.2 \%)$ & 0.146 \\
\hline \multicolumn{4}{|l|}{ Anticoagulation } \\
\hline Aspirin & $572(51.7 \%)$ & $553(50.0 \%)$ & 0.444 \\
\hline Other & $535(48.3 \%)$ & $554(50.0 \%)$ & \\
\hline Symptomatic VTE & $15(1.36 \%)$ & $37(3.34 \%)$ & 0.003 \\
\hline Pulmonary emboli & $1(0.09 \%)$ & $12(1.08 \%)$ & 0.005 \\
\hline Deep vein thrombosis & $15(1.36 \%)$ & $28(2.53 \%)$ & 0.065 \\
\hline
\end{tabular}

Table 6. Patient characteristics, comorbidities, operative factors and type of VTE prophylaxis for the matched revision TJA cohort and VTE rates. BMI Body Mass Index, $\mathrm{Kg}$ kilogram, $M$ meter, CCI Charlson Comorbidity Index, $C H F$ congestive heart failure, $C P D$ cardiopulmonary disease, $C V D$ cardiovascular disease, $D M$ diabetes mellitus, $M I$ myocardial infraction, $P U D$ peptic ulcer disease, $P V D$ peripheral vascular disease, $V T E$ venous thromboembolism event. ${ }^{*}$ The groups were matched for age, BMI, sex, all comorbidities in the cohort (see Table 1), joint, unilateral vs. bilateral, as well as the use of cement and TXA.

of our study may help to raise awareness on the substantial increase in VTE risk following a blood transfusion, thereby prompting greater efforts to ration the use of transfusions and reduce VTE rates. For this purpose, Stanford Hospital recently implemented an online system that required clinicians to review the guidelines for transfusion before approving a blood transfusion order ${ }^{34}$. This simple reminder decreased the number of blood transfusions at the hospital by $24 \%$, resulting in improved patient outcomes and reduced annual costs. In addition to greater awareness, additional blood management strategies should be employed for TJA with the aim of reducing the need for blood transfusion. These efforts may encompass pre-operative identification of patients at high risk for transfusion, correcting pre-operative anemia with haemopoietic agents, salvaging blood lost during the peri-operative period, limiting post-operative blood loss with haemostatic measures and individualizing transfusion triggers according to a patient's symptoms and medical co-morbidities ${ }^{35}$.

Results from our regression analysis also suggested that aspirin may have a protective role in both primary and revision TJA. However, several issues prevent us from making this conclusion; first, aspirin prophylaxis has gradually become the gold standard at our institution thus more aggressive anticoagulants were most likely provided to patients that were at higher risk. Our main effort was to investigate the independent association of blood transfusion with VTE and our adjustments and matching were done accordingly thus we cannot make 
conclusions about the effect chemoprophylaxis agents had on VTE rates. Second, all non-aspirin prophylaxis agents were grouped under one umbrella. Dichotomization to an aspirin and non-aspirin group may introduced further bias to the independent effect aspirin has on VTE. Finally, As opposed to blood transfusion rates that remained constant during the past decade, aspirin and TXA have gradually increased together with a decrease in VTE event rates (Fig. 1). Confounders related to advancement in care such as earlier walking, same day physiotherapy and better pain control that cannot be adjusted for may have played a role. While these changes may have added to a decrease in overall VTE rates thus limiting our conclusions regarding aspirin and TXA, blood transfusion rates did not change during the course of this study therefore we believe these unmeasurable changes had a negligible effect on our conclusion about blood transfusions.

This study is not without limitations. First, this was a retrospective study which relied on data from medical records and coding for analysis and therefore conclusion making. The retrospective nature of this study may also had led to inadvertent inaccuracies in data collection as well as missing variables ${ }^{36}$. Due to the large scale study over a long period of time we were unable to contact patients for verification and complementary purposes. Second, VTE is effected by multiple genetic and environmental causes and many risk factors have been proposed ${ }^{37-39}$. While all efforts were made to capture and adjust for a comprehensive range of variables, unmeasurable variables as well as measurable variables that were not routinely recorded such as genetic predisposition and family history of VTE may have played a role in VTE development and hence confounded the results ${ }^{37}$. Third, despite the implementation of a nurse navigator program to track the perioperative course of all patients undergoing TJA at our institution ${ }^{40}$, it is possible that some VTE events may have gone undetected or unrecorded, especially if treated in an outside facility. This is especially true for DVT for which there is a higher possibility for events to either be undetected or for events to be captured without our ability to adequately assess clinical severity and this may have resulted in underestimating the association between blood transfusion and DVT as opposed to our findings on PE. Furthermore, as with previous VTE studies, event rate was relatively low effecting the magnitude of our findings ${ }^{5,19}$. It also prevented us from examining PE and DVT as independent outcomes in a regression analysis as the maximum likelihood estimation of the logistic model would suffer from small-sample bias ${ }^{41}$. Finally, in the absence of a definite institutional protocol for blood transfusions, we were unable to control for the differing indications for receiving a blood transfusions and could not comment on the necessity of transfusions recorded in this study. The use of blood transfusions at our institution was relatively low compared prior literature ${ }^{11}$, possibly affecting the generalizability of our results.

In conclusion, our findings show a three times higher rate of VTE in patients who receive blood transfusions following TJA. This association was mainly due to higher symptomatic PE rates. Greater efforts should be made to educate surgeons about this dangerous association and to develop perioperative protocols to reduce the need for blood transfusions in order to mitigate this risk.

Received: 26 May 2021; Accepted: 5 October 2021

Published online: 28 October 2021

\section{References}

1. Zhang, Z. et al. Risk factors for venous thromboembolism of total hip arthroplasty and total knee arthroplasty: A systematic review of evidences in ten years. BMC Musculoskelet. Disord. 16, 24. https://doi.org/10.1186/s12891-015-0470-0 (2015).

2. Parvizi, J., Huang, R., Rezapoor, M., Bagheri, B. \& Maltenfort, M. G. Individualized risk model for venous thromboembolism after total joint arthroplasty. J. Arthroplasty 31, 180-186. https://doi.org/10.1016/j.arth.2016.02.077 (2016).

3. Porter, S. B., White, L. J., Osagiede, O., Robards, C. B. \& Spaulding, A. C. Tranexamic acid administration is not associated with an increase in complications in high-risk patients undergoing primary total knee or total hip arthroplasty: A retrospective casecontrol study of 38,220 patients. J. Arthroplasty 35, 45-51.e3. https://doi.org/10.1016/j.arth.2019.08.015 (2020).

4. Goel, R. et al. Association of perioperative red blood cell transfusions with venous thromboembolism in a North American Registry. JAMA Surg. 153, 826-833. https://doi.org/10.1001/jamasurg.2018.1565 (2018).

5. Acuña, A. J., Grits, D., Samuel, L. T., Emara, A. K. \& Kamath, A. F. Perioperative Blood transfusions are associated with a higher incidence of thromboembolic events after TKA: An analysis of 333,463 TKAs. Clin. Orthop. Relat. Res. 479(3), 589-600 (2021).

6. Jackson, A. et al. Association of perioperative red blood cell transfusion with symptomatic venous thromboembolism following total hip and knee arthroplasty. J. Arthroplasty 36(1), 325-330 (2021).

7. Shander, A. et al. Activity-based costs of blood transfusions in surgical patients at four hospitals. Transfusion 50(4), 753-765 (2010).

8. Jankite, M. K. Blood transfusion: Cost, quality, and other considerations for the surgical management of the critically ill. Crit. Care Nurs. Q. 42, 173-176. https://doi.org/10.1097/CNQ.0000000000000250 (2019).

9. Shander, A. \& Goodnough, L. T. Can blood transfusion be not only ineffective, but also injurious?. Ann. Thorac. Surg. 97, 11-14. https://doi.org/10.1016/j.athoracsur.2013.08.007 (2014).

10. Trends in United States blood collection and transfusion: Results from the 2013 AABB Blood Collection, Utilization, and Patient Blood Management Survey. Google Search. https://www.google.com/search?q=Trends+in+United+States+blood+collection+and+trans fusion $\% 3 \mathrm{~A}+$ results +from+the+2013+AABB+blood+collection $\% 2 \mathrm{C}+$ utilization $\% 2 \mathrm{C}+$ and + patient+blood+management+survey $\&$ $\mathrm{oq}=$ Trends+in+United+States+blood+collection+and+transfusion $\% 3 \mathrm{~A}+$ results + from + the $+2013+\mathrm{AABB}+\mathrm{blood}+$ collection $\% 2 \mathrm{C}+$ utilization\%2C+and+patient+blood+management+survey\&aqs=chrome..69i57.548j0j7\&sourceid=chrome\&ie=UTF-8 $($ accessed April 20, 2021).

11. Browne, J. A., Adib, F., Brown, T. E. \& Novicoff, W. M. Transfusion rates are increasing following total hip arthroplasty: Risk factors and outcomes. J. Arthroplasty 28, 34-37. https://doi.org/10.1016/j.arth.2013.03.035 (2013).

12. Song, K., Pan, P., Yao, Y., Jiang, T. \& Jiang, Q. The incidence and risk factors for allogenic blood transfusion in total knee and hip arthroplasty. J. Orthop. Surg. Res. 14, 273. https://doi.org/10.1186/s13018-019-1329-0 (2019).

13. Frank, S. M. et al. Variability in blood and blood component utilization as assessed by an anesthesia information management system. Anesthesiology 117, 99-106. https://doi.org/10.1097/ALN.0b013e318255e550 (2012).

14. Soleimanha, M. et al. A survey on transfusion status in orthopedic surgery at a trauma center. Arch. Bone Joint Surg. 4, 70-74 (2016). 
15. Spencer, J., Thomas, S. R. Y. W., Yardy, G., Mukundan, C. \& Barrington, R. Are we overusing blood transfusing after elective joint replacement? A simple method to reduce the use of a scarce resource. Ann. R. Coll. Surg. Engl. 87, 28-30. https://doi.org/10.1308/ 1478708051379 (2005)

16. Overuse of Blood Transfusions Can Negatively Impact Surgical Patients. Lippincott's Bone Joint Newsl. 18, 91-92. https://doi.org/ 10.1097/01.BONEJ.0000419359.77339.3a. (2012).

17. Khera, R. et al. Adherence to methodological standards in research using the national inpatient sample. JAMA 318, $2011-2018$. https://doi.org/10.1001/jama.2017.17653 (2017).

18. Teng, T. L., Menendez, M. E., Okike, K., Cassidy, C. \& Salzler, M. Most orthopaedic studies using the national inpatient sample fail to adhere to recommended research practices: A systematic review. Clin. Orthop. Relat. Res. https://doi.org/10.1097/CORR. $0000000000001355(2020)$.

19. Jiang, T., Song, K., Yao, Y., Pan, P. \& Jiang, Q. Perioperative allogenic blood transfusion increases the incidence of postoperative deep vein thrombosis in total knee and hip arthroplasty. J. Orthop. Surg. Res. 14, 235. https://doi.org/10.1186/s13018-019-1270-2 (2019).

20. von Elm, E. et al. Strengthening the reporting of observational studies in epidemiology (STROBE) statement: Guidelines for reporting observational studies. BMJ 335, 806-808. https://doi.org/10.1136/bmj.39335.541782.AD (2007).

21. R Core Team. R: A Language and Environment for Statistical Computing. (R Foundation for Statistical Computing, 2020) https:// www.R-project.org/.

22. Byrnes, J. R. \& Wolberg, A. S. Red blood cells in thrombosis. Blood J. Am. Soc. Hematol. 130(16), 1795-1799 (2017).

23. Valles, J. et al. Erythrocytes metabolically enhance collagen-induced platelet responsiveness via increased thromboxane production, adenosine diphosphate release, and recruitment. Blood 78, 154-162. https://doi.org/10.1182/blood.V78.1.154.154 (1991).

24. Lowe, G. D., Lee, A. J., Rumley, A., Price, J. F. \& Fowkes, F. G. Blood viscosity and risk of cardiovascular events: The Edinburgh Artery Study. Br. J. Haematol. 96, 168-173. https://doi.org/10.1046/j.1365-2141.1997.8532481.x (1997).

25. Holley, L., Woodland, N., Hung, W. T., Cordatos, K. \& Reuben, A. Influence of fibrinogen and haematocrit on erythrocyte sedimentation kinetics. Biorheology 36, 287-297 (1999).

26. Rampling, M. W. The binding of fibrinogen and fibrinogen degradation products to the erythrocyte membrane and its relationship to haemorheology. Acta Biol. Med. Ger. 40, 373-378 (1981).

27. Byrnes, J. R. \& Wolberg, A. S. Red blood cells in thrombosis. Blood 130, 1795-1799. https://doi.org/10.1182/blood-2017-03-745349 (2017).

28. Walton, B. L. et al. Elevated hematocrit enhances platelet accumulation following vascular injury. Blood 129, 2537-2546. https:// doi.org/10.1182/blood-2016-10-746479 (2017).

29. Bilgin, Y. M. \& Brand, A. Transfusion-related immunomodulation: A second hit in an inflammatory cascade?. Vox Sang. 95 , 261-271. https://doi.org/10.1111/j.1423-0410.2008.01100.x (2008).

30. Hod, E. A. Red blood cell transfusion-induced inflammation: Myth or reality. ISBT Sci. Ser. 10, 188-191. https://doi.org/10.1111/ voxs.12108 (2015).

31. Silvain, J. et al. Impact of red blood cell transfusion on platelet aggregation and inflammatory response in anemic coronary and noncoronary patients: The TRANSFUSION-2 study (impact of transfusion of red blood cell on platelet activation and aggregation studied with flow cytometry use and light transmission aggregometry). J. Am. Coll. Cardiol. 63, 1289-1296. https://doi.org/10. 1016/j.jacc.2013.11.029 (2014).

32. Schisterman, E. F., Cole, S. R. \& Platt, R. W. Overadjustment bias and unnecessary adjustment in epidemiologic studies. Epidemiology $20(4), 488$ (2009).

33. national_summit_overusepdf.pdf.

34. Goodnough, L. T. et al. Improved blood utilization using real-time clinical decision support. Transfusion 54, 1358-1365. https:// doi.org/10.1111/trf.12445 (2014).

35. Liu, D., Dan, M. \& Adivi, N. Blood conservation strategies in total hip and knee arthroplasty. Reconstr. Rev. 4(4), 39-39 (2014).

36. Sedgwick, P. Retrospective cohort studies: Advantages and disadvantages. The BMJ. https://www.bmj.com/content/348/bmj.g1072 (accessed September 14, 2021).

37. Anderson, F. A. \& Spencer, F. A. Risk factors for venous thromboembolism. Circulation 107, 1-9. https://doi.org/10.1161/01.CIR. 0000078469.07362.E6 (2003).

38. Mantilla, C. B., Horlocker, T. T., Schroeder, D. R., Berry, D. J. \& Brown, D. L. Risk factors for clinically relevant pulmonary embolism and deep venous thrombosis in patients undergoing primary hip or knee arthroplasty. Anesthesiology 99, 552-560. https://doi.org/ 10.1097/00000542-200309000-00009 (2003).

39. Klarin, D. et al. Genome-wide association analysis of venous thromboembolism identifies new risk loci and genetic overlap with arterial vascular disease. Nat. Genet. 51, 1574-1579. https://doi.org/10.1038/s41588-019-0519-3 (2019).

40. A Nurse Navigator Program Is Effective in Reducing Episode-of-Care Costs Following Primary Hip and Knee Arthroplasty. https:// pubmed.ncbi.nlm.nih.gov/31130443/ (accessed April 20, 2021).

41. King, G. \& Zeng, L. Logistic regression in rare events data. Polit. Anal. 9, 137-163 (2001).

\section{Author contributions}

N.S. and Ja.P. conceptualized the study. N.S., L.L., G.G., and J.P. collected the data. M.S. analyzed the data. M.S. and N.S. prepared the figures. N.S., L.L., G.G., M.S., and J.P. wrote the manuscript. Ja.P. edited the manuscript. All authors reviewed and approved the final version of the manuscript.

\section{Competing interests}

The authors declare no competing interests.

\section{Additional information}

Supplementary Information The online version contains supplementary material available at https://doi.org/ 10.1038/s41598-021-00263-0.

Correspondence and requests for materials should be addressed to J.P.

Reprints and permissions information is available at www.nature.com/reprints.

Publisher's note Springer Nature remains neutral with regard to jurisdictional claims in published maps and institutional affiliations. 
(c) (i) Open Access This article is licensed under a Creative Commons Attribution 4.0 International cc) License, which permits use, sharing, adaptation, distribution and reproduction in any medium or format, as long as you give appropriate credit to the original author(s) and the source, provide a link to the Creative Commons licence, and indicate if changes were made. The images or other third party material in this article are included in the article's Creative Commons licence, unless indicated otherwise in a credit line to the material. If material is not included in the article's Creative Commons licence and your intended use is not permitted by statutory regulation or exceeds the permitted use, you will need to obtain permission directly from the copyright holder. To view a copy of this licence, visit http://creativecommons.org/licenses/by/4.0/.

(C) The Author(s) 2021 